\title{
Ueber Störungen im Gebiete der Sinnesperception bei Geisteskranken.
}

\author{
Von
}

\author{
Professor Dr. W. v. B E CH T E R EW.
}

Jene psychischen Störungen, die zu Herabsetzung oder Steigerung der Sinnesperception vcier zum Auftreten selbstständiger Empfindungen elementarer Art - wie Schmerzen oder sonstiger abnormer Empfindungen - Anlass geben, bleiben in dem allgemeinen Symptomencomplex sicher nicht ohne Bedeutung. In vieler Hinsicht indessen sind sie noch nicht genügend erforscht, weshalb hier auf den Gegenstand eingegangen werden darf $\left.{ }^{1}\right)$.

Die fraglichen Erscheinungen erinnern in gewissem Sinne an physische Veränderungen der Sensibilität oder gewöhnliche Anästhesien, Hyperästhesien oder Neuralgien, unterscheiden sich von ihnen aber pathogenetiseh dadurch, dass sie bei Geisteskranken nicht durch irgendwelche Störungen der Leitungsfunctionen des Nervensystems hervorgerufen werden, sondern durch den psychischen Zustand der Kranken bedingt werden. Sie erscheinen also als psychisch bedingte Störungen der Empfindungen und können zum Unterschied von jenen als psychische Anästhesien, psychische Hyperästhesien und psychische Algien oder kürzer Psycho-anästhesien, Psycho-hyperästhesien und Psycho-algien bezeichnet werden.

Psycho-anästhesien äussern sich durch mehr oder weniger deutliche Abschwächung der Sinnesperception und können ebenso sehr im Gebiete der allgemeinen Sensibilität, wie in der Sphäre der Sinnesorgane beobachtet werden. Bedingt sind sie entweder durch allgemeines Sinken des Bewusstseins bei Geisteskranken oder aurch Ablenkung ihrer Aufmerksamkeit infolge von krankhaften Erscheinungen. Als Prototyp der ersteren erscheint in normalem Zustande jene Schwächung der Sinnesperception, die bei Gesunden während des Schlummers oder Schlafes beobachtet wird, als Prototyp der letzteren jene Abschwächung der Sinnesperception, die bei jedem Gesunden im Falle der Ablenkung der Aufmerksamkeit zur Beobachtung gelangt. Bei angestrengter geistiger Arbeit ist Betäubung quälender Zahnschmerzen nichts ungewöhnliches. In der Hitze des Gefechtes oder im Handgemenge weiss der Kämpfende nichts von seinen Wunden.

1) Kurz hingewiesen wird auf ähnliche Störungen bei Ebbinghaus, Kraftt-Ebing und anderen. 
Ganz analoge Erscheinungen kommen nicht selten auch bei Geisteskranken zur Beobachtung. So bei den schlafähnlichen Zuständen, wie sie z. B. im Verlaufe der $\Lambda$ mentia Meynert's oder bei gewissen epileptischen Formen des Irreseins beobachtet werden, erweist sich die Gefühlsperception deutlich herabgesetzt, so dass äussere Rejze auf die Hautoberfläche oder Eindrücke auf die Sinnesorgane nicht mit der gleichen Schärfe, wie in normalem Zustande, zur Apperception gelangen.

Dies ergiebt olne weiteres die unmittelbare Beobachtung am Krankenbette und die Prüfung ihrer kutanen Sensibilitiit; endlich weisen auch die Kranken selbst in einzelnen Fällen bei Besserung ihres Zustandes auf allgemeine Abstumpfung ihrer Sensibilitätsperception hin.

Sind andererseits die Kranken mit ihrem Wahn beschäftigt, ist ihre Aufmerksamkeit durch die cine oder die andere Idee beschäftigt, so nimmt die Sinnesperception dabei naturgemäss $\mathrm{ab}$ und tritt eine mehr oder weniger deutlich ausgesprochene Psycho-anästhesie in die Erscheinung. Seinen Höhepunkt erreicht dieser Zustand während der Exstase bei religiöser Erregung, allein er ist auch unter anderen Verhältnissen zu beobachten, wenn die Aufmerksamkeit der Kranken durch reichliche akustische, optische oder andere Halluzinationen abgelenkt ist.

In einigen Fälen nehmen diese Psycho-anlisthesien systematischen Charakter an; sie treten dann in Beziehung zu irgend einer besonderen Art von Empfindurgen auf.

So giebt es Psycho-anästhesien im Gebiete des Gemeingefühls. Sie sind $n$. a bei Hysterie nichts seltenes, doch kommen sie anch im Verlaufe anderer, besonders organischer. Psychosen zur Beobachtung. Solche Kranke klagen manchmal selbst über Leere im Kopf, dass sie ilıen Kopf, ihren Körper, ihre Eingeweide nicht fühlen, obwohl die spezielle Prüfung der Sensibilität der Haut und der tieferen Teile des Körpers keine wesentlichen Verändicrungen ergiebt.

Es versteht sich von selbst, dass derartige Psycho-anästhesien in gewissen Fällen zur Entwicklung der merkwürdigen Wahnvorstellung vom Fehlen der Eingeweide, vom Eintritt des Todes u. s. w. hinführen.

Folgender Fall von Katatonie bietet ein klassisches Beispiel von hochgradig entwickelter Psycho-anästhesie im Gebiete des Gemeingefühls.

Es handelt sich um ein 20jähriges junges Mädchen, Jüdin, Tochter eines Kaufmanns, aus gesunder Familie. Schwere Nerven- oder Geisteskrankheiten sind in der Verwandtschaft nicht vorhanden. Vater 60 a. n., gesund. Mutter, 50 a. n., leidet manchmal an starkem Herzklopfen. Grossvater mütterlicherseits $90 \mathrm{a}, \mathrm{n}$., gesund. Grossmutter mütterlicherseits 88 a.n., gesund. Grossvater und Grossmutter väterlicherseits sind in hohem Greisenalter unter unbekannten Umständen gestorben. Patientin ist das siebente, jüngste Kind ihrer Eltern. Sie hat zwei gesunde Brüder und vier Schwestern, von denen die älteste eine sehr nervöse, reizbare Person ist. Die Kinder ihrer Schwestern und Brüder sind gesund. 
Bis zu ihrem ersten Lebensjahre war Patientin durchaus gesund. Im Laufe des zweiten bekam sie ein Geschwür am rechten Unterschenkel. Das Leiden dauerte zwei Jahre. Es wurde zweimal operiert. Patientin lahmt seitdem ihr ganzes Leben und ist, befürchtend lächerlich zu erscheinen, gezwungen sich vom Verkehr mit Menschen zurückzuziehen. Sie war immer niedergeschlagen und reizbar. Eine andere Krankheit ausser der genannten hat bis zum 16. Jahr nicht bestanden. Vom 17. Lebensjahre leidet Patientin an Blntarmut und starkem Herzklopfen. Vor zwei Jahren stellte sich ein Augenleiden ein, znerst rechts, dann links. Das Augenleiden war chronisch mit häufigen Exacerbationen, von starken Schmerzen begleitet, Die letzte Exacerbation war im Frühjahr 1901. Die Yatientin haite damals die Befürchtung zu erblinden. Im Mai 1902 kommt sie aus Kiew nach St. Petersburg, wm ihr Augenleiden spezialistisch behandeln zu lassen. Um diese Zeit verschlimmerte sich auch ihr nervöser Zustand. Starke Schwitche, hitufiges Erbrechen, ein Globusgefühl im Halse stellte sich ein. Sie bekam subkutane Arseninjektionen Anfang Juni trat hinzu hartnaickige Sclilaflosigkeit, Schwermut, Unruhe: die Patientin sagte, sie fülsle ihren Kopf, ihren Mund nicht, sie wisse nicht, ob sie satt sei, 11. s. W. In letzter Zeit ist eine Verschlimmerung eingetreten. Die Kranke hat die Forstellung, sie sei tot.

Patientin untermittelgross, von schlechtem Eraihrungszustand. Gesichtsfarbe erdtahl. Sichtbare Schleimhäute leicht anämisch mit Ausnahme der Conjunctiva beider Augen, die hochgradig hyperämisch ist. Muskulatur mïssig entwickelt. Knochensystem regelrecht entwickelt, mit Ausnahme der rechten Tibia, die rauh erscheint und etwas empfindlich bei der Palpation. Auf der Aussenfliche des rechten Unterschenkels eine lineare Narbe herrïhrend von einer Sequestrotomie. Antlit\% ohne Asymmetrien. Linke Pupille wegen hinterer Synechien verengt. Spuren von abgelanfener Iritis an beiden Augen. Rechte Pupille reagiert gut, linke etwas schwicher, Beweglichkeit beider Augäplel normal. In iereflexe leicht gesteigert, links etwas mehr wis rechts. Selmenreflexe der Oberextremititen deutlich ansgesprochen, gleichmaissig auf beiden Seiten. Schluckreflex links und rechts erhalten. Schmerzempfindlichkeit unverändert. Mnskelkraft herabgesetzt, Gang rechts lihmend. Herztöne rein. Puls 76 , regelmassig, mässig gespannt. Abdomen eingezogen, Wandungen schlaff. Hartnaickige Stuhlverstopfung. In Urin weder Eiweis, noch Zncker.

Das 'Antlit\% der Patientin hat den Ausdruck quillender Unruhe. Sie sient beständig angstroll tum sich, sitzt fortwihhend auf ihrem Lager, das Kinn auf die linke Hand gestiitat. Mit klighlicher Stimme bittet sie den Ar\%, ihr zu helfen und zwar unweigerlich soiort; fragt, ob sie heilbar, und fleht um Rettung, da ihr sonst Untergang drohe. Sie ist von ihren Ideen vollständig absorbiert, eriasst nur mühsam Fragen, die sich nicht auf das Gebiet ihres Wahnes beziehen: sie liisst sie entweder ganz unbeantwortet oder tut dies mit Unterbrechungen, un Hilfe. Rettung u. s. w. bittend. Die Krauke behauptet, es scheine ihr, sie labe keinen Kopf, keine Hinde, keine Füsse, in ihrer Mitte sei es leer u. s. w. Hin und wieder erklärt sie, ihr liopf sei vom Herzen getrennt, _durch Krankheit nach hinten gedreht" und ganz verrückt. Sie hat keine Gedanken, kein Gadächtnis, keine W ansche, keine Freude, keine Tratuer. Sie behauptet, sie könne weder gehen, noch stehen, noch liegen, noch sit\%en, und doch steht sie, dazu aufgefordert, sofort auf, geht hin und her u. s, w. Peinvoll ist ihr das Bewusstsein der Enmöglichkeit, etwas zu tun oder zu wïnschen. Sie behauptet, nicht essen zu kőnnen, keinen Hunger zu fühlen, die Speisen nicht wahruehmen zu köunen. Wird sie \%um Essen angeregt, gefütert, so nimmt sie gentigend Nahrung $\%$ sich. Sie versichert, keinen Schlaf finden zu künnen. Es scheint ihr, dass sie stark abgemagert sei, dass nur dic Knochen zurückgeblieben, dass sie anch die Knochen nicht fühlt, dass ihr Zustand von Tag zu Tag sich verschlimmert, dass sie unheilbar ist. Sie glaubt, ihre jetzige Krankheit rühre davon her, dass ihr Augenleiden mit Finreibungen von Quecksilber behandelt wurde. Patientin hiilt bestaindig ihren Kopf, um sicher zu sein, dass sie ihn noch hat, und doch ist ihr, 
als habe sie keinen Kopf, als fühle sie ihn nicht. Sie behauptet, in ihrem Körper habe nur noch die Zunge allein Leben. Sie denkt "mit Hilfe der Zunge“, fürchtet sich „mit der Zunge“, wünscht mittelst der Zunge u. s. w. Wenn sie zu reden aufhört, so hört damit ihr Leben auf, sie wird wie „eine Tote“.

Bei der Untersuchung und durch Befragen wird eruiert, dass die Sensibilität (Tast-, Schmerz-, Muskelsensibilität) bei der Kranken vollständig erhalten ist. Sie giebt selbst zu, sie fühle ihren Körper und Kopf, wenn sie sie mit der Hand berührt, und nichtsdestoweniger ist ihr, als hätte sie weder Kopf noch Rumpf; sie fühlt sie nicht, wenn sie auch überzeugt ist, dass sie vorhanden sind. Druck auf die Baucheingeweide empfindet die Kranke ebenfalls, und doch fühlt sie sie selbst nicht. So ist es auch mit den Händen: es scheint der Patientin, dass sie nicht vorhanden sind, während sowohl die kutane, wie die tiefe Sensibilität der Hände (Muskeln, Knochen, Bänder) völlig erhalten ist. Patientin ist überzeugt, dass sie Hände besitzt, nur glaubt sie, dass sie körperlos sind, bewegt sie in Gegenwart dritter Personen hin und her, um sich von ihrer Schwere zu überzeugen.

Patientin behauptet auch, nicht zu fühlen, dass sie esse, dass sie satt werde u. s. w. Sie empfindet nicht Leid noch Freud; ihr Aechzen und Stöhnen komme nur "von der Zunge“.

Wenn sie ihr Kinn" fortwährend auf die Hand stützt. so giebt dies ihr, wie Patientin erklärt, zwar nicht die Empfindung des Kopfes, aber sie weiss dann wenigstens, wo ihr Kopf sich befindet. Mit der Zeit hat sich ein starrer, zeitweise kataleptischer Zustand bei der Kranken entwickelt, verbunden mit dem Gedanken, sie sei bereits tot, und begleitet von totaler Stummheit und Nahrungsverweigerung. Nach einigen Wochen löst sich dieser Zustand von Starre mit allmählichem Uebergang in Besserung.

In der betreffenten Entwicklungsphase der Krankheit handelt es sich also unzweifelhaft um Anästhesie im Gebiete des Allgemeingefühls, doch ist diese, wie die Untersuchung ergiebt, nicht physischer, sondern psychischer Art.

Es versteht sich nun von selbst, dass eine derartige Anästhesie, wie im vorliegenden Falle, zu entsprechenden Wahnideen führen kann als Fehlen der Eingeweide, Eintritt des Todes u. s. w. Es erscheint bemerkenswert, dass neben psychischer Anästhesie bei unserer Kranken auch psychische Anästhesie des seelischen Empfindens vorhanden war. Obwohl Patientin augenscheinlich leidet und ihr Leid durch Aechzen, Stöhnen, mimische Bewegungen und Bitten um Hilfe zum Ausdruck bringt, behauptet sie demungeachtet, sie fühle nicht Leid, noch Freude und sei unfähig sie zu fühlen, und wenn sie um Hilfe bitte, so geschehe dies nur mit der Zunge (d. h. mit Worten). Etwas ähnliches kommt bekanntlich bei gewissen schweren Melancholieformen zur Beobachtung.

Was den entgegengesetzten Zustand, den der psychischen Hyperästhesie betrifft, so wird derselbe im Gegensatze zu den psychischen Anästhesien bedingt entwoder durch eine allgemeine Steigerung aller psychischen Processe und deutlichere Ausprägung derselben, oder aber durch specielle Konzentration der Aufmerksamkeit auf bestimmte Eindrücke. Derartige Hyperästhesien kommen im Gebiete der Hautsensibilitait und der Sinnesorgane zur Beobachtung bei maniakalischer Erregung, sowie bei 
allen Zuständen psychischer Erregung, hier vorzugsweise bedingt durch Steigerung psychischer Prozesse.

Bei primärem Irresein kommen infolge des systematischen Wahnzustancies und der bestehenden Hallucinationen in der Regel psychische Hyperästhesien zur Beobachtung unter dem Einfluss der Konzentration der Aufmerksamkeit auf einen bestimmten Ideenkreis. Diese Hyperästhesien erscheinen also von systematischer Art.

Als Beispiel derartiger systematischer psychischer Hyperästhesien kann ich einen von mir behandelten Officier anführen, der im russisch-türkischen Krieg eine Kontusion erhielt und seitdem an traumatischer Neurose mit Geistesstörung leidet. Er vertrug nicht das Wort „Blut“ und zeigte eine auffallende Hyperästhesie gegenüber allem Roten. Bei dem Worte Blut und dem Anblick eines roten Gegenstandes geriet er in heftige Aufregung und bekam echte Jirämpfe. Psychische Hyperästhesien im Gebiete des Gemeingefühls sind bei Geisteskrankheiten ebenfalls nicht selten; sie äussern sich als allgemeine Zerschlagenheit, Steigerung des Hunger- und Sattgefühls u. s. w. Eine besondere Bedeutung aber erlangen psychische Hyperästhesien bekanntlich bei hypochondrischen Zuständen und dem hypochondrischen Irresein, wo die in der Norm undeutlich empfundenen inneren Organe nicht nur deutlich von den Kranken gefühlt werden, sondern sie äusserst belästigen.

Nicht zu leugnen ist natürlich, dass die Ursache der Hyperästhesie der inneren Organe bei diesen Zuständen nicht selten in bestimmten Affektionen dieser Organe (Magendarmkatarrh, Stauungen im Pfortadersystem u. s. w.) zu suchen ist, wobei es also gewissermassen um eine Hyperästhesio physischer Art sich handeln würde. Zweifellos ist aber auch, dass neben einer solchen, physischen oder Leitungs-Hyperästhesie bei jenen Zuständen auch psychische Hyperästhesien im Gebiete des Gemeingefühls zur Beobachtung kommen; denn ein derartiger Kranker braucht nur an seine Eingeweide zu denken, um sofort bestimmte Empfindungen in ihnen auszulösen. Dass auf Grund dieser psychischen Hyperästhesien Wahnideen über Fäulnis und Zerfall der Eingeweide etc. sich entwickeln können, ist so allgemein bekannt, dass darauf nicht näher eingegangen zu werden braucht.

Von wesentlicher Bedeutung ist nun natürlich die Frage, wie wir psychische Anästhesien oder Hyperästhesien von physischen oder Leitungs-Anästhesien bezw. - Hyperästhesien unterscheiden sollen. Soweit meine eigenen Erfahrungen in dieser Beziehung reichen, erscheint als wichtiges unterscheidendes Merkmal das Eehlen irgendwelcher Hinweise auf Störungen des Leitungsvermögens im Nervengewebe, die eine physikalische oder Leitungs-Anästhesie bezw. -Hyperästhesie bedingen könnten; weiterhin ihr allgemeiner oder systematischer Charakter und schliesslich ihre Abhängigkeit von dem Zustand der Psyche und das damit zusammenhängende Schwanken ihrer Intensität je nach den Veränderungen des Seelenzustandes. 
Abgesehen von psychischen Anästhesien und psychischen Hyperästhesien giebt es bei Geisteskranken noch eine Reihe von kranlshaften Empfindungen psychischen Ursprungs, die als psychische Algien oder Psychoalgien bezeichnet werden können. Es handelt sich um Schmerz- oder andere Empfindungen in verschiedenen Körperteilen und Eingeweiden bei Geisteskranken, grösstenteils von vager Art, im engsten Zusammenhang mit dem Seelenzustande der betreffenden Kranken. Bei Bestehen von Halluzinationen, wie z. B. bei dem chronischer halluzinatorischen Irresein, gewinnen diese Psychoalgien nicht selten innigste Wechselbeziehungen zu Gehörs- und anderen Halluzinationen, von denen sie gewissermassen suggestiv erregt werden, indem sie ihrerseits als Folgeerscheinung Halluzinationen auslösen. Besonders typische Beispiele verschiedener Psychoalgien bieten uns Kranke, die an chronischem halluzinatorischen Irresein auf dem Boden von Alkoholismus leiden. Einer meiner Kranken dieser Art, den ich noch während meiner Lehrtätigkeit in Kasan in dem dortigen Bezirkshospital behandelte, und der an beständigen zablreichen Gehörshalluzinationen litt, hatte die allermannigfaltigsten psychoalgischen Empfindungen von Nadelstichen, Brennen u. s. w. in bestimmten Körperteilen.

In der Regel waren die psychoalgetischen Empfindungen dieses Kranken verbunden mit Gehörshalluzinationen, wobei er die Worte „wir einen Klystier machen den Rheumatismus!“ zu vernehmen glaubte, sodass jedesmal, wenn der Kranke Schmerz in einem bestimmten Körperteil empfand, er zugleich den Ruf „wir einen Klystier den Rheumatismus“ vernahm, was seiner Ansicht nach bedeutete, dass das Stechen und Brennen von Personen herrühre, deren Stimmen er hörte. $\mathrm{Zu}$ bemerken ist, dass bei diesem Kranken die Sensibilität und Motilität keinerlei objektive Veränderungen darbot und auch die inneren Organe gesund waren.

Bei einer anderen Patientin, die an chronischem halluzinatorischen Irresein mit zahlreichen Gehörshalluzinationen leidet und einige Zeit im Krankenhause des Heil. Nicolaus des Wunderthäters behandelt wurde, bestand der Wahn, dass die Aerzte der Anstalt mit dem Oberarzte an der Spitze auf spiritistischem Wege Ungehörigkeiten, Quälereien und Foltern an ihr. ausübten. Unterstützt wurde dieser Wahn durch entsprechende Gehörshalluzinationen (Stimmen der Anstaltsärzte), die bei ihr in auffallender Weise mit verschiedenen psychoalgischen Empfindungen in äusseren und inneren Körperteilen vergesellschaftet waren. Die Kranke führt ein genanes Tagebuch über ihre Empfindungen und halluzinatorischen Erscheinungen; für ihren Zustand charakteristisch sind einige Auszïge aus den umfangreichen Memoiren der Kranken, die sie mir in der Hoffinung überliess, durch mich von ihren lästigen Peinigern befreit zu werden.

- Hier z. B. einige Stellen aus den Aufzeichnungen der Kranken vom 8. Oktober 1901: 
„Im rechten Knie krampfhafte heftige Schmerzen, im linken Knie Bohren und heftige Schmerzen, so dass ich nicht gehen noch aufstehen kann. Im rechten $\mathrm{Ohr}$ Stechen, starker gewaltsamer Schmerz, Stechen in den Hühneraugen, Stechen durch Brust und Rücken auf der linken Seite unweit vom Herzen, starke Schmerzen im linken Knie, Winde aus dem Harnorgan, starke Schlaflosigkeit, ein unbegreifliches Gefühl in der Brust, ein gemeines, mit Angst, etwas leidenschaftliches - sinnliches, Winde aus dem Harnorgan mit den Worten: „nicht der A . . . allein giebt klangvolle Winde, sondern auch die P.... und S.... (vulgäre Bezeichnungen für Vulva und Penis). Schmerzen im linken Knie, Schmerzen im rechten Knie mit den Worten: „du verzeichnest Schmerzen im linken Knie, also soll es im rechten Knie weh tun, wie?- Kitzeln in den weichen Teilen des Geschlechtsorgans mit den Worten: -wenn es juckt, so kratze dich! hast du denn keine Hände, was? Oder nehmen einen Stock und reiss die P.... auf" (vulgäre Bezeichnung für Vulva). Lachreiz in dèr Brust, starkes Herzklọpfen während zwei Minuten, nach Ausspritzung stark eitriges Wasser, gemeines Gefühl im Harnorgan mit den Worten: „unsere Wärterinnen (d. h. vom Hospital Nibolai d. Wundertaters) lieben dieses Gefühl, protestieren nicht dagegen, weshalb also sollte es dir nicht gefallen?" (folgt ein schmutziges Schimpfwort).

9. Oktober. Die ganze Nacht Klopfen im Magen, wio mit einem Hammer, Stechen in der rechten Hinterbacke, starkes Klopfen in der Gebärmutter, legt man die Hand auf diese Stelle, so erhebt es sich darunter mit den Worten: "lege die Hand nicht an und es wird sich nichts rühren". Von der Ferse bis zum Nacken durch die ganze Wirbelsäule ein Tönen in den Knochen, als wenn mit einer grossen Schraube etwas in den Knochen hineingeschraubt würde, Stechen unter dem rechten Auge, Stich in die rechte Hinterbacke, dufregung im ganzen Eingeweide, Selbstgefühl sehr schlecht, Zittern im ganzen Körper, Schmerz im linken Knie, Harndrang, eitriger Urin mit den Worten: "geh pissen, aber wische dich nicht unten, wir trocknen alles selbst durch Hitze" u. s. w. Stechen in den weichen Teilen des Geschlechtsorgans, als wenn jemand mit Nadeln hineinstechen würde; Winde aus den Geschlechtsteilen mit den Worten: „schreibe doch auf, wie das Geschlechtsorgan heisst!" u. s. w.

Am 11. Oktober schreibt die Kranke: Im rechten Schulterblatt krampfhafter Schmerz und Stechen; Stich ins Knie. Stich in die Fusssohle; Stich in den rechten Augapfel mit den Worten: „du bist uns zuwider, seit man dich nachts im Hemde in die Anstalt brachte, bist du uns durch dein Aeusseres zuwider geworden; grosser Bauch, kurzer Rumpf, lange Beine, dünnes Haar, unordentliche Frisur, Körper unrein, daher es bequem ist, mit dir alle möglichen Schweinereien vorzunehmen, dich zu verspotten; du sassest wie betrunken oder benommen, wie ein wahres Luder!" u.s. w,

In einem weiteren Heft rom 19. Oktober schreibt die Kranke unter anderem: Stiche in die Sehne der linken Hand, starkes Klopfen im Magen, Schmerzen in der rechten Seite mit den Worten: „man muss die Leber befühlen" (folgt ein zotiges Schimpfwort). Schütteln im ganzen Eingeweide, Stechen in den weichen Teilen des Körpers, in den Geschlechtsteilen, Urindrang, eitriger Urin, Stechen in den After mit den Worten: "nicht wahr, wie mit einer Nadel!".

Ar einer andern Stelle unter dem 27. Oktober hört die Kı Stichen in den After bereits die Stimme: , du musst wissen, was Hämorrhoiden sind, um zu begreifen, wie Hämorrhoiden gewonnen werden!"

Aus den mehr als 20 Heften, die die Kranke mir übergab, könnten noch unendlich viele ähnliche Stellen mitgeteilt werden, doch wird das Angeführte genügen, um zu zeigen, wie eng die verschiedenen psychoalgischen Empfindungen mit den Gehörshalluzinationen der Kranken verflochten sind.

Von neuralgischen Schmerzen unterscheiden sich diese psychoalgischen Empfindungen dadurch, dass sie ungemein ver- 
breitet erscheinen, dass sie durch höchste Mannigfaltigkeit ihres Charakters und grosse Veränderlichkeit auffallen, dass sie in direkter Abhängigkeit von dem Seelenzustande des betreffenden Kranken stehen, und endlich durch jene Wechselbeziehungen zu krankhaften Störungen des Bewusstseins bezw. Halluzinationen, die wir soeben kennen lernten. 